Check for updates

The BMJ

Cite this as: BMJ 2021;374:n1950 http://dx.doi.org/10.1136/bmj.n1950 Published: 4 August 2021

\title{
Covid-19: CPAP reduces need for invasive mechanical ventilation in patients requiring oxygen, study finds
}

\section{Elisabeth Mahase}

Continuous positive airway pressure (CPAP) reduced the need for invasive mechanical ventilation in adults admitted to hospital with acute respiratory failure due to covid-19, a trial has found. ${ }^{1}$

The Recovery Respiratory Support trial compared CPAP, high flow nasal oxygenation (HFNO), and conventional oxygen therapy among inpatients with covid-19 who required oxygen therapy, looking at the rates of tracheal intubation or mortality within 30 days with each treatment.

A preprint of the findings has reported that neither CPAP nor HFNO, when compared with conventional oxygen therapy, reduced mortality at any point.

During the first peak of covid-19 in the UK, $80.4 \%$ of patients admitted to hospital received supplementary oxygen. This fell to around 50\% from July. ${ }^{2}$ Both CPAP and HFNO are used for patients with other respiratory diseases such as community acquired pneumonia, but there were concerns that they might only delay tracheal intubation in patients with covid-19 while also exacerbating lung injury.

\section{Outcomes}

In the study, which was funded by the UK National Institute for Health Research, 1272 patients from around the UK were randomly allocated to one of the three delivery systems from April 2020 to May 2021.

Looking at a composite outcome of intubation or death within 30 days of randomisation, the study reported that the need for tracheal intubation or mortality was lower in the CPAP group (36.3\%) than in the conventional oxygen therapy group (44.4\%). However, the researchers said that the decrease seen among the CPAP group was "driven by a decrease in the incidence of tracheal intubation, with no statistically significant difference in rate of 30 day mortality."

Fewer participants in the CPAP group required admission to critical care, and in those who required tracheal intubation the time to intubation was longer.

No difference was found between HFNO (44.4\%) and conventional oxygen therapy $(45.1 \%)$ for the composite outcome.

Speaking at a Science Media Centre briefing, Danny McAuley, the study's chief investigator and an intensive care medicine consultant at the Royal Victoria Hospital in Belfast, said, "CPAP reduces the pressure on the need for invasive mechanical ventilation and the pressure on intensive care unit beds. HFNO uses a large amount of oxygen and can cause issues with limited oxygen therapy, and we've found that it doesn't really add anything above conventional therapy."
He said that the findings may be beneficial in systems with limited resources, such as oxygen.

The researchers noted a number of limitations to the trial, including that they did not achieve the planned sample size owing to the decision to stop recruitment because of practical reasons linked to reducing cases of covid-19 in the UK, as well as an ethical obligation to share accumulated data with the international clinical community.

1 Perkins GD, Ji C, Connolly BA, et al. An adaptive randomized controlled trial of non-invasive respiratory strategies in acute respiratory failure patients with COVID-19. medRxiv 2021 [preprint]. https://www.medrxiv.org/content/10.1101/2021.08.02.21261379v1.

2 Docherty AB, Mulholland RH, Lone NI, etallSARIC4C Investigators. Changes in in-hospital mortality in the first wave of COVID-19: a multicentre prospective observational cohort study using the WHO Clinical Characterisation Protocol UK. Lancet Respir Med 2021;9:773-85. https://www.thelancet.com/journals/lanres/article/PIIS2213-2600(21)001752/fulltext. doi: 10.1016/S2213-2600(21)00175-2 pmid: 34000238

This article is made freely available for use in accordance with BMJ's website terms and conditions for the duration of the covid-19 pandemic or until otherwise determined by BMJ. You may use, download and print the article for any lawful, non-commercial purpose (including text and data mining) provided that all copyright notices and trade marks are retained. 